\title{
Somatic Segregation in Diploid Chlamydomonas reinhardii
}

\author{
By R. F. MATAGNE* AND A. ORBANS \\ Laboratory of Molecular Genetics, Department of Botany, \\ University of Liège, B-4000 Liège, Belgium
}

(Received 3 September 1979; revised 9 November 1979)

\begin{abstract}
A double heterozygous arg-7-7 $+/+$ pab-2 diploid strain of Chlamydomonas reinhardii, phenotypically wild-type, was treated with $N$-methyl- $N^{\prime}$-nitro- $N$-nitrosoguanidine. A total of 5500 colonies isolated on rich medium from the mutagenized cells were replicated to minimal medium. Nine auxotrophs $(0 \cdot 16 \%)$ were isolated: six required $p$-aminobenzoic acid, two required arginine and one required both $p$-aminobenzoic acid and nicotinamide. On the basis of their cell volume, mating-type and DNA content, all the auxotrophs appeared to be diploids. However, analysis of crosses between the auxotrophs and the haploid wild-type strain suggested that the auxotrophs are probably aneuploids. This conclusion is also supported by the slow growth, heterogeneity in colony size and reduced pigmentation of certain of the auxotrophs cultivated on supplemented medium. The same conclusion is supported by control experiments in which $0 \cdot 10 \%$ auxotrophs were recovered. One auxotroph, however, apparently resulted from mitotic crossing-over at the four-strand stage. Under our experimental conditions, the mutagen does not seem to play a significant role in the induction of somatic segregation.
\end{abstract}

\section{INTRODUCTION}

In the green alga Chlamydomonas reinhardii, the diploid phase is normally restricted to the zygote which, after maturation, undergoes meiosis and germinates to produce four or eight haploid progeny cells. However, a small fraction of the newly formed zygotes divides mitotically to produce vegetative diploid cells (Ebersold, 1967). Such diploids are easily selected by crossing complementary auxotrophic haploid mutants and plating the zygotes on minimal medium. We previously used this complementation test to analyse the fine structure of the arg-7 cistron and to study gene-enzyme relationships in this system (Loppes et al., 1972; Matagne, 1976, 1978).

In the experiments described here, we treated a double heterozygous diploid strain $\operatorname{arg-7-7}+/+p a b-2$ with the mutagen $N$-methyl- $N^{\prime}$-nitro- $N$-nitrosoguanidine (NTG) in an attempt to isolate dominant auxotrophic mutations. We recovered only $\mathrm{Arg}^{-}, \mathrm{Pab}^{-}$and $\mathrm{Nic}^{-}$strains. The results of subsequent genetical analysis indicated that no dominant mutations had been induced. Moreover, in control experiments in which no mutagen was used, we found that auxotrophs could also be recovered at a similar rate. The data are therefore most easily explained by assuming that most of the isolated $\mathrm{Arg}^{-}$and $\mathrm{Pab}^{-}$auxotrophs result because of aneuploidization and are not induced by the mutagen.

\section{METHODS}

Strains. All strains used in this study were derived from the $137 \mathrm{c}$ wild-type strain (WT) of Chlamydomonas reinhardii. The double heterozygous diploid arg-7-7 $+/+p a b-2$ was obtained by crossing the haploid arg-7-7 (an arginine-requiring mutant mapping in the arg-7 cistron) mating-type plus ( $\mathrm{mt}^{+}$) strain with the haploid $p a b$-2 (requiring $p$-aminobenzoic acid) mating-type minus $\left(\mathrm{mt}^{-}\right)$strain; a triple heterozygous diploid 
$+a r g-7-7+/ a c-14+p a b-2$ was isolated by crossing the haploid arg-7-7 strain with the haploid $a c-14 p a b-2$ strain (requiring both acetate and $p$-aminobenzoic acid for growth). These three markers are located in linkage group I and the relevant map distances are: centromere to $a c-14,12$ units; $a c-14$ to $\arg -7-7,4$ units; arg-7-7 to pab-2, 15 units (Levine \& Goodenough, 1970). Both the double and triple heterozygous diploid strains are phenotypically wild-type and are normally maintained on $\mathbf{M}$ medium.

Media. The following media were used: $\mathrm{M}$ or $400 \mathrm{~N}$, basal minimal medium containing $400 \mathrm{mg} \mathrm{NH}_{4} \mathrm{Cl} \mathrm{l}^{-1}$ (Loppes, 1966); $20 \mathrm{~N}+$ arg, minimal medium containing only $20 \mathrm{mg} \mathrm{NH}_{4} \mathrm{Cl} \mathrm{l}^{-1}$ and $50 \mathrm{mg}$ arginine $1^{-1}$; $\mathrm{M}-\mathrm{N}+\mathrm{YE}$, minimal medium lacking $\mathrm{NH}_{4} \mathrm{Cl}$ and supplemented with $4 \mathrm{~g}$ Difco yeast extract $\mathrm{l}^{-1}$. In supplemented media, $p$-aminobenzoic acid (pab) or nicotinamide (nic) were added at $1 \mathrm{mg} \mathrm{l}^{-1}$ and sodium acetate at $0.5 \mathrm{~g}^{-1}$. Media were solidified by adding 15 or $40 \mathrm{~g}$ Difco agar $1^{-1}$ to the solutions.

Mutagenic treatment. Diploid cells $\left(7 \times 10^{7}\right)$ grown in $\mathrm{M}$ liquid medium under 8000 lux white light were centrifuged and resuspended in $15 \mathrm{ml}$ NTG solution $\left(100 \mu \mathrm{g} \mathrm{ml}^{-1}\right.$ in $0.03 \mathrm{M}$-potassium phosphate buffer, $\mathrm{pH} 7.0)$ at $25^{\circ} \mathrm{C}$. After treatment, the cells were washed with similar phosphate buffer and plated on $\mathrm{M}-\mathrm{N}+\mathrm{YE}$ medium at a dilution subsequently yielding 50 to 200 colonies per plate.

Determination of cell volumes and DNA contents. The auxotrophic strains, recovered following mutagenesis and maintained on $\mathrm{M}-\mathrm{N}+\mathrm{YE}$ agar medium, were suspended in distilled water overnight in the dark to aid in the dissociation of clumped cells and to increase homogeneity in cell size (R. F. Matagne, unpublished observation). This treatment also induces gametogenesis. A Coulter counter was used to determine the size distribution of this population of gametes (Loppes et al., 1972).

For DNA determinations, these gametes were centrifuged $(900 \mathrm{~g}, 5 \mathrm{~min})$ and the pellets were fixed in absolute ethanol at $0^{\circ} \mathrm{C}(2 \mathrm{~h})$. The fixed cells were washed once with absolute ethanol and then with water, treated with ribonuclease $\left(1 \mathrm{mg} \mathrm{ml}^{-1}\right)$ at $37^{\circ} \mathrm{C}$ for $1 \mathrm{~h}$, and then washed once with water and stained with the fluorescent dye propidium iodide $\left[0.05 \mathrm{mg} \mathrm{ml}^{-1}\right.$ in $1 \%(\mathrm{w} / \mathrm{v})$ sodium citrate]. DNA measurements were made using a flow cytofluorometer (Ortho Instruments-FC-200, equipped with an argon laser $480 \mathrm{~nm}$, $50 \mathrm{~mW}$ ). For each strain, 20000 to 60000 cells were analysed.

Genetic analysis. Crosses and maturation of zygotes were carried out according to the method of Levine \& Ebersold (1958). After germination of the zygotes on $4 \%$ agar $M$ or $M-N+Y E$ medium, the spores were collected at random and plated on $M-N+Y E$ medium after appropriate dilution. The resultant colonies were replica-plated to $M$ or to appropriate enriched media.

\section{RESULTS}

\section{Isolation and analysis of auxotrophs after NTG treatment}

In a preliminary experiment, the survival of the diploid strain $\arg -7-7+/+p a b-2$ was determined after exposure to NTG for various periods. For the induction of mutations, treatment with NTG for $45 \mathrm{~min}$, giving a survival of about $10 \%$, was chosen. Ten days after treated cells had been plated on $\mathrm{M}-\mathrm{N}+\mathrm{YE}$ medium, the resultant colonies were replicated to $M$ medium and to fresh $M-N+Y E$ medium. Out of 5500 replicated colonies, nine auxotrophs unable to grow on $\mathrm{M}$ medium were isolated. They were analysed for growth on different selective media: six required $p$-aminobenzoic acid $\left(\mathrm{Pab}^{-}\right)$, two required arginine $\left(\mathrm{Arg}^{-}\right)$and one was a double mutant requiring both $p$-aminobenzoic acid and nicotinamide $\left(\mathrm{Pab}^{-} \mathrm{Nic}^{-}\right)$.

To explain the origin and the nature of these nine strains, three simple hypotheses can be proposed. (1) The $\mathrm{Arg}^{-}$and $\mathrm{Pab}^{-}$auxotrophs are diploid mutants which have become homozygous for the arg-7 or pab-2 marker by NTG-induced mutation at the corresponding wild-type locus ( $\arg -7-7+/$ arg-7-x pab-2 and $\arg -7-7$ pab- $2 /+$ pab-2; arg-7-x and arg-7-7 being non-complementing mutants of the $\arg -7$ locus). The Nic - mutant resulted from an additional dominant mutation at a nic locus. (2) The Arg- and $\mathrm{Pab}^{-}$auxotrophs arose by haploidization or aneuploidization of certain diploid cells allowing expression of these recessive markers on chromosome I; the nic mutation would be the only mutation (dominant if on a disomic chromosome in the aneuploid, recessive if in a haploid cell or on a monosomic chromosome in the aneuploid) induced by NTG. (3) The auxotrophs are diploids in which the $\mathrm{Nic}^{-}$phenotype resulted from a dominant induced mutation while the $\mathrm{Arg}^{-}$ and $\mathrm{Pab}^{-}$phenotypes arose by mitotic recombination, i.e. crossing-over at the four-strand stage, or gene conversion of the $\arg -7^{+}$and $p a b-2^{+}$alleles to $\arg -7$ and $p a b-2$, respectively.

To distinguish between these hypotheses, we have analysed some additional properties 
Table 1. Cell volume and relative cell DNA content of the WT haploid, arg-7-7 + / + pab-2 diploid and nine auxotrophs

Cell volumes were determined by Coulter counter measurements; DNA contents were measured by flow cytofluorometry (mean of two measurements).

$\begin{array}{clcc}\begin{array}{c}\text { Strain } \\ \text { Haploid WT }\end{array} & \begin{array}{c}\text { Volume } \\ \left(\mu \mathrm{m}^{3}\right)\end{array} & \begin{array}{c}\text { Relative DNA } \\ \text { content }\end{array} \\ \text { Diploid } & \text { Wild-type } & 25( \pm 5) & 1 \cdot 0 \\ 1 & \text { Wild-type } & 51( \pm 10) & 2 \cdot 1 \\ 2 & \text { Pab }^{-} & 49( \pm 10) & 2 \cdot 4 \\ 3 & \text { Arg- }^{-} & 62( \pm 10) & 2 \cdot 6 \\ 4 & \text { Arg- }^{-} & 57( \pm 10) & 2 \cdot 3 \\ 5 & \text { Pab-Nic- }^{-} & 53( \pm 10) & 1 \cdot 9 \\ 6 & \text { Pab- }^{-} & 51( \pm 10) & 2 \cdot 0 \\ 7 & \text { Pab }^{-} & 53( \pm 10) & 2 \cdot 1 \\ 9 & \text { Pab- }^{-} & 56( \pm 10) & 2 \cdot 4 \\ 9 & \text { Pab- }^{-} & 53( \pm 10) & 2 \cdot 0 \\ & \text { Pab- }^{-} & 51( \pm 10) & 2 \cdot 2\end{array}$

Table 2. Phenotypes of the progeny produced by crosses between the nine auxotrophs and the haploid WT

Zygotes were matured and germinated on $\mathrm{M}$ medium except in two cases $\left(^{*}\right)$ where they were plated on $\mathrm{M}-\mathrm{N}+\mathrm{YE}$.

\begin{tabular}{|c|c|c|c|c|}
\hline Cross & $\begin{array}{l}\text { Phenotype of } \\
\text { isolate }\end{array}$ & $\begin{array}{c}\text { No. of } \\
\text { colonies } \\
\text { analysed }\end{array}$ & $\begin{array}{l}\text { No. of } \\
\text { auxotrophs } \\
\text { recovered }\end{array}$ & $\begin{array}{l}\text { Phenotypes and no. } \\
\text { of auxotrophs recovered }\end{array}$ \\
\hline $1 \times \mathrm{WT}$ & $\mathrm{Pab}^{-}$ & 406 & 18 & All $\mathrm{Pab}^{-}$ \\
\hline $2 \times \mathrm{WT}$ & $\mathrm{Arg}^{-}$ & 133 & 8 & All Arg- \\
\hline $3 \times$ WT & Arg- & 67 & 5 & All Arg- \\
\hline $4 \times \mathrm{WT}$ & $\mathrm{Pab}^{-} \mathrm{Nic}^{-}$ & 310 & 23 & $7 \mathrm{Pab}^{-}, 10 \mathrm{Nic}^{-}, 6 \mathrm{Pab}^{-} \mathrm{Nic}^{-}$ \\
\hline $4 \times W^{*}$ & $\mathrm{Pab}^{-} \mathrm{Nic}^{-}$ & 350 & 34 & $14 \mathrm{Pab}^{-}, 18 \mathrm{Nic}^{-}, 2 \mathrm{Pab}^{-\mathrm{Nic}^{-}}$ \\
\hline $5 \times \mathrm{WT}$ & $\mathrm{Pab}^{-}$ & 130 & 6 & All $\mathrm{Pab}^{-}$ \\
\hline $6 \times \mathrm{WT}$ & $\mathrm{Pab}^{-}$ & 133 & 10 & All $\mathrm{Pab}^{-}$ \\
\hline $6 \times W^{*}$ & $\mathrm{Pab}^{-}$ & 294 & 58 & All $\mathrm{Pab}^{-}$ \\
\hline $7 \times$ WT & $\mathrm{Pab}^{-}$ & 14 & 1 & All $\mathrm{Pab}^{-}$ \\
\hline $8 \times$ WT & $\mathrm{Pab}^{-}$ & 90 & 12 & All $\mathrm{Pab}^{-}$ \\
\hline $9 \times$ WT & $\mathrm{Pab}^{-}$ & 55 & 10 & All $\mathrm{Pab}^{-}$ \\
\hline
\end{tabular}

of the isolated auxotrophs. According to hypotheses 1 and 3, the auxotrophs must be diploids, whereas in hypothesis 2, they are haploids or aneuploids. In Chlamydomonas, diploids are mating-type minus ( $m t^{-}$is dominant to $m t^{+}$; Ebersold, 1967) and have a larger cell volume than haploid cells (Loppes et al., 1972); moreover, crosses between the arg-7-7 + /arg-7-x pab-2 or + pab-2/arg-7-7 pab-2 diploids and the wild-type haploid strain should give rise to $\mathrm{Arg}^{-} \mathrm{Pab}^{-}$haploid segregants from the triploid zygotes.

The mating-type of each of the nine auxotrophic strains was determined; all were found to be $m t^{-}$. The mean cell volume of the haploid WT strain was $25 \mu \mathrm{m}^{3}$; the auxotrophs had mean cell volumes not significantly different from the original diploid strain $\left(51 \mu \mathrm{m}^{3}\right)$ (Table 1). The DNA content per cell was determined by cytofluorometry of cell suspensions of the auxotrophs (Table 1). The observed values indicated that the auxotrophs have a DNA content similar to, or even higher than, that of the reference diploid. On the basis of these observations, the auxotrophs appear to be diploids.

In an attempt to recover $\mathrm{Arg}^{-} \mathrm{Pab}^{-}$haploid segregants, the nine auxotrophs were crossed to the haploid WT $\left(m t^{+}\right)$strain. Certain crosses (especially strain $7 \times \mathrm{WT}$ ) yielded very low germination frequencies. Thus, in some cases, very few zygote progeny clones could be analysed even though several hundred zygotes had been recovered. The results of the 
genetic analyses are summarized in Table 2. The Pab- strains (1, 5, 6, 7, 8 and 9) when crossed with WT produced only WT and Pab- progeny, whereas the Arg- strains (2 and 3) crossed with WT gave rise to WT and Arg- segregants only. In the cross, isolate 4 $\left(\mathrm{Pab}^{-} \mathrm{Nic}^{-}\right) \times \mathrm{WT}$, single $\mathrm{Pab}^{-}$or $\mathrm{Nic}^{-}$isolates as well as double mutants $\left(\mathrm{Pab}^{-} \mathrm{Nic}^{-}\right)$ were recovered.

In addition to the $68 \mathrm{Pab}^{-}$segregants recovered in the cross, isolate $6 \times \mathrm{WT}, 19$ colonies with unexpected phenotypes were isolated. Three of these grew slowly on $M$ medium but grew normally on $M$ medium supplemented with $p$-aminobenzoic acid. The 16 other segregants did not grow either on minimal or on $20 \mathrm{~N}+$ arg medium, grew slowly on $\mathrm{M}+$ pab and showed normal growth on rich complete medium as well as on $\mathrm{M}+\mathrm{pab}+$ acetate. These phenotypes are distinctly different from either parental type.

As the germination of the zygotes was routinely induced on $4 \%$ agar $\mathrm{M}$ medium before plating the spores on rich medium, it could be argued that this procedure might lead to selection against certain auxotrophs, especially the double mutant Arg-Pab-. Additional crosses were therefore performed with strains 4 and 6 and the zygotes were matured and germinated in $\mathrm{M}-\mathrm{N}+\mathrm{YE}$ medium: the results (Table 2) indicated that the nature of the germination medium did not affect the types of auxotrophs recovered.

To determine whether the nic mutation is dominant or recessive, a double haploid (or aneuploid) mutant $\mathrm{Pab}^{-} \mathrm{Nic}^{-}\left(\mathrm{mt}^{+}\right)$isolated from the cross, isolate $4 \times \mathrm{WT}$, was crossed with $\arg -7\left(m t^{-}\right)$and the immature zygotes were plated on $\mathrm{M}+$ nic medium; after $5 \mathrm{~d}$, colonies (diploid or aneuploid) formed by complementation between the arg-7 pab-2 loci were obtained. Further analyses showed that these colonies could grow in the absence of nicotinamide, thus proving that the nic mutation is recessive.

On the basis of these genetical analyses, it appears that the auxotrophs resulted neither from induced mutation nor from conversion of the wild-type alleles to $\arg -7-7$ or $p a b-2$, respectively. Mitotic crossing-over between the two markers is also an unlikely explanation since no double mutant $\mathrm{Arg}^{-} \mathrm{Pab}^{-}$was recovered from any of the crosses. The $\mathrm{Nic}^{-}$phenotype is apparently the only one resulting from NTG-induced mutation. It is known that in Chlamydomonas, nic and ac (acetate) mutations are the most frequent types isolated after mutagenic treatment of haploid cells (Loppes, 1970; Schimmer \& Loppes, 1975).

To compare the growth of the NTG-induced auxotrophs with the growth of haploid and diploid control cells, the different strains were plated at low cell densities (50 to 300 cells per plate) on M-N+YE agar medium. Three haploid strains (WT, $\operatorname{arg-7-7}$ and pab-2) and two diploid strains (the parental strain arg-7-7 $+/+p a b-2$ and an arginine-requiring diploid ac-14 arg-7-7 $+/+$ arg-7-7 pab-2) were used as controls. Colony sizes were compared after $10 \mathrm{~d}$ growth. The auxotrophic isolates 1 and 9 formed large colonies homogeneous in size, not significantly different from the five control strains. Other isolates produced small (isolates 4 and 6) or tiny (isolates 2, 3 and 5) colonies rather heterogeneous in size. Two isolates $(7$ and 8 ) produced minute and very pale green colonies. Some colonies of isolates 3 and 6 were also pale green.

\section{Recovery of auxotrophs in control experiments}

To see if the recovery of auxotrophs from diploid prototrophic cells was related to the treatment with the mutagen, we performed control experiments in which diploid cells were plated on $\mathrm{M}-\mathrm{N}+\mathrm{YE}$ agar medium without previous exposure to NTG. The colonies formed after $10 \mathrm{~d}$ were replicated to $\mathrm{M}$ and to fresh $\mathrm{M}-\mathrm{N}+\mathrm{YE}$ medium.

In the first experiment, the diploid $\arg -7-7+/+p a b-2$ was used; out of 8000 colonies analysed, five $\mathrm{Pab}^{-}$mutants were recovered. These mutants were not studied further. In a second experiment, we analysed 10500 colonies from the triple heterozygous diploid + arg-7-7 +/ac-14 + pab-2. After replica-plating to $\mathrm{M}$ and to $\mathrm{M}-\mathrm{N}+\mathrm{YE}$ medium, followed by testing on individually supplemented media, eight $\mathrm{Arg}^{-}$, one $\mathrm{Pab}^{-}$and five $\mathrm{Pab}^{-} \mathrm{Ac}^{-}$auxotrophs were recovered. Colony sizes for these spontaneous auxotrophs grown 
Table 3. Phenotypes of the auxotrophic progeny recovered from crosses between the spontaneous $\mathrm{Arg}^{-}, \mathrm{Pab}^{-} \mathrm{Ac}^{-}$and $\mathrm{Pab}^{-}$auxotrophs and haploid $\mathrm{WT}$

\begin{tabular}{|c|c|c|}
\hline Cross & $\begin{array}{l}\text { No. of } \\
\text { auxotrophs } \\
\text { recovered }\end{array}$ & $\begin{array}{l}\text { Phenotype and } \\
\text { segregation }\end{array}$ \\
\hline $\begin{array}{l}\mathrm{Arg}-\times \mathrm{WT} \\
\mathrm{Pab}^{-} \mathrm{Ac}^{-} \times \mathrm{WT} \\
\mathrm{Pab}^{-} \times \mathrm{WT}\end{array}$ & $\begin{array}{l}28 \\
43 \\
41\end{array}$ & $\begin{array}{l}\text { All } \mathrm{Arg}^{-} \\
42 \mathrm{Pab}^{-} \mathrm{Ac}^{-}: 1 \mathrm{Pab}^{-} \\
24 \mathrm{Pab}^{-} \mathrm{Ac}^{-}: 17 \mathrm{Pab}^{-}\end{array}$ \\
\hline
\end{tabular}

on agar-solidified $\mathrm{M}-\mathrm{N}+\mathrm{YE}$ were compared with those of the haploid and diploid wildtype strains. Here also, some strains (among them the $\mathrm{Pab}^{-}$auxotroph) produced large colonies homogeneous in size and similar in this respect to the wild-type strains, whereas others formed colonies more heterogeneous in size.

Three auxotrophic isolates (one $\mathrm{Arg}^{-}$, one $\mathrm{Pab}^{-} \mathrm{Ac}^{-}$and one $\mathrm{Pab}^{-}$) were crossed to the haploid WT $\left(m t^{+}\right)$cells. To study their genetical background, the zygotes were matured on $\mathrm{M}-\mathrm{N}+\mathrm{YE}$ medium and the progeny were analysed on selective media (Table 3 ). In the cross $\mathrm{Arg}^{-} \times \mathrm{WT}$, only $\mathrm{Arg}^{-}$segregants were recovered, suggesting that the $a c-14 \mathrm{pab}-2$ markers were no longer carried by the parental strain. Conversely, in the cross $\mathrm{Pab}^{-} \mathrm{Ac}^{-} \times \mathrm{WT}$, $42 \mathrm{Pab}^{-\mathrm{Ac}^{-}}$and one $\mathrm{Pab}^{-}$auxotroph were obtained in the progeny, indicating the absence of the arg-7-7 marker in the parent. Finally, the cross $\mathrm{Pab}^{-} \times \mathrm{WT}^{\prime}$ gave rise to $\mathrm{Pab}^{-} \mathrm{Ac}^{-}$ and $\mathrm{Pab}^{-}$segregants in a ratio not significantly different from $1: 1$. This suggests that the $\mathrm{Pab}^{-}$parent was genotypically $++p a b-2 / a c-14+p a b-2$.

\section{DISCUSSION}

After mutagenic treatment of the diploid strain arg-7-7 $+/+$ pab-2 with NTG, we obtained nine auxotrophic isolates (two $\mathrm{Arg}^{-}$, six $\mathrm{Pab}^{-}$and one $\mathrm{Nic}^{-}$) out of 5500 colonies analysed. Analysis of their mating-type, cell volume and DNA content indicates that they are clearly not haploid, but could be diploid. Genetical analysis indicates that the $\mathrm{Arg}^{-}$or $\mathrm{Pab}^{-}$auxotrophs do not correspond to the genotypes arg-7-7 + / arg-7-x pab-2 or arg-7-7 $p a b-2 /+p a b-2$ since no double mutant $\mathrm{Arg}^{-} \mathrm{Pab}^{-}$was recovered from any of the crosses between the auxotrophs and a WT haploid strain. The hypothesis of mutation at the wildtype locus corresponding to the arg-7 or the $p a b-2$ locus (hypothesis 1) can thus be eliminated. For the same reason, the conversion of the wild-type allele to the corresponding mutant allele (hypothesis 3 ) can also be ruled out. Furthermore, the results cannot be explained by mitotic crossing-over between the arg and pab markers. A crossing-over between the arg marker and the centromere could result in the observed auxotrophic phenotype (which would be genotypically arg-7-7 +/arg-7-7 + or + pab-2/+pab-2). However, one would still expect some isolates to be $\arg -7-7 p a b-2 /+p a b-2$ due to crossing-over between the two markers since the map distance from the centromere to $\operatorname{arg-7-7}$ (16 units) is the same as that between $a r g-7-7$ and $p a b-2$ (as far as one can extrapolate from meiotic recombination to mitotic recombination).

The simplest explanation should be that at least some auxotrophs result from the loss of chromosome I or a segment of it, thereby allowing expression of the recessive markers carried on the homologous chromosome. The nic mutation, which is recessive, apparently arose from the mutagenic action of NTG at a nic locus with the simultaneous loss of the corresponding wild-type allele.

The same interpretation can be applied to certain of the auxotrophs (Arg- and $\mathrm{Pab}^{-} \mathrm{Ac}^{-}$) recovered in the control experiment in which the diploid + arg-7-7 + /ac-14 + pab-2 parent was not exposed to NTG. The genetical analysis of two of these spontaneous auxotrophs, one $\mathrm{Arg}^{-}$and one $\mathrm{Pab}^{-} \mathrm{Ac}^{-}$, indicated that they were bearing only arg-7-7 or 
pab-2 ac-14 markers, respectively. However, the $\mathrm{Pab}^{-}$strain isolated in the same experiment apparently is of the genotype $++p a b-2 / a c-14+p a b-2$ : this suggests mitotic crossingover between the markers $a c-14$ and $\arg -7-7$ at the four-strand stage giving a chromatid bearing the alleles $a c-14^{+} \arg -7-7^{+} p a b-2(++p a b-2)$.

An additional argument in favour of the hypothesis of aneuploidization is the slow and heterogeneous growth of some of the auxotrophs. In Aspergillus, aneuploids, which occur spontaneously at high frequency $(1 \%$ or more; Käfer, 1960,1961$)$, are often poor in vigour as compared to euploids. An unbalanced chromosome complement could be responsible for the heterogeneity in size of colonies and abnormally low pigmentation of some strains.

In an abstract, Martinek et al. (1970) reported mitotic segregation from diploid strains of Chlamydomonas; they proposed as a mechanism for these segregations either nondisjunction or mitotic crossing-over.

Lee et al. (1976) reported the isolation of aneuploids from diploid cells, on the basis of DNA content intermediate between $\mathrm{n}$ and $2 \mathrm{n}$ and the loss of heterozygosity at different loci. The aneuploids represented about $1 \%$ of the colonies scored and were induced after $6 \mathrm{~h}$ of $\mathrm{NH}_{4}+$ starvation followed by incubation in the dark for $6 \mathrm{~d}$. Other auxotrophs isolated by these authors were interpreted as being mitotic recombinants. In later experiments, R. W. Lee (personal communication) recovered auxotrophs (less than $1 \%$ of the colonies scored) without any special conditions and found no significant differences between the frequencies of auxotrophs arising under standard conditions as compared to conditions of nitrogen and light starvation.

Finally, the results reported here $(0 \cdot 10 \%$ auxotrophs in the control, $0 \cdot 16 \%$ after NTG treatment) indicate that the frequencies of auxotrophs recovered with or without mutagenic treatment do not differ significantly. Thus, under our experimental conditions, the mutagen does not seem to play a significant role in the induction of somatic segregation. In Aspergillus, Käfer (1960) found that nitrogen mustard or radiation could increase the frequency of somatic segregation.

It should be noted that the frequencies of aneuploidization reported here involve only one chromosome pair. If aneuploids are produced by a chance mechanism, the actual frequencies of aneuploidization would be: $0 \cdot 10$ to $0 \cdot 16 \% \times 16$, i.e. $1 \cdot 6$ to $2 \cdot 5 \%$. These high frequencies, similar to those reported in Aspergillus (Käfer, 1960), have to be taken into account when experimenting with diploid Chlamydomonas cells.

We gratefully acknowledge the help of Mrs A. Miller-Faures (Laboratory of Molecular Biochemistry, University of Mons) who made the DNA determination by flow cytofluorometry. Professor Robert W. Lee (Department of Biology, Dalhousie University) is also specially thanked for his constructive discussion of the manuscript.

\section{REFERENCES}

EBERSOLD, W. T. (1967). Chlamydomonas reinhardi: heterozygous diploid strains. Science 157, 447449.

KÄFER, K. (1960). High frequency of spontaneous and induced somatic segregation in Aspergillus nidulans. Nature, London 186, 619-620.

KÄFER, E. (1961). The processes of spontaneous recombination in vegetative nuclei of Aspergillus. Genetics 46, 1581-1609.

LeE, R. W., Whiteway, M. S. \& Yorke, M. A. (1976). Recovery of sexually viable non-diploids from diploid Chlamydomonas reinhardtii. Genetics 83 (suppl.), 44.

Levine, R.P. \& Ebersold, W. T. (1958). Gene recombination in Chlamydomonas reinhardi. Cold
Spring Harbor Symposia on Quantitative Biology 23, 101-109.

LeVine, R. P. \& Goodenough, U. W. (1970). The genetics of photosynthesis and of the chloroplasts in Chlamydomonas reinhardi. Annual Review of Genetics 4, 397-408.

LoPPES, R. (1966). Damage induced by methyl methanesulfonate (MMS) in Chlamydomonas reinhardi. Zeitschrift für Vererbungslehre 98, 193202.

LOPPES, R. (1970). Selection of arginine-requiring mutants in Chlamydomonas reinhardi after treatment with three mutagens. Experientia 26, 660661.

Loppes, R., Matagne, R. F. \& StriJkert, P. J. 
(1972). Complementation at the arg-7 locus in Chlamydomonas reinhardi. Heredity 28, 239-251.

MartineK, G. W., Ebersold, W. T. \& Nakamura, K. (1970). Mitotic recombination in Chlamydomonas reinhardi. Genetics 64 (suppl.), 41.

Matagne, R. F. (1976). Arg-7 mutant $\times$ wild-type crosses in Chlamydomonas reinhardi: study of the enzyme produced in diploid strains. Molecular and General Genetics 146, 209-214.
Matagne, R. F. (1978). Fine structure of the arg-7 cistron in Chlamydomonas reinhardi. Complementation between arg-7 mutants defective in argininosuccinate lyase. Molecular and General Genetics 160, 95-99.

SCHIMMER, O. \& LOPPES, R. (1975). Forward mutations induced by nitrosoguanidine during the synchronized cell cycle of Chlamydomonas reinhardi. Molecular and General Genetics 138, 25-31. 